\title{
First-principles calculations that clarify energetics and reactions of oxygen adsorption and carbon desorption on $4 \mathrm{H}-\mathrm{SiC}(11 \overline{2} 0)$ surface
}

\author{
Han Li, ${ }^{1}$ Yu-ichiro Matsushita, ${ }^{1}$ Mauro Boero, ${ }^{2}$ and Atsushi Oshiyama ${ }^{1}$ \\ ${ }^{1}$ Department of Applied Physics, The University of Tokyo, Hongo, Tokyo, 113-8656, Japan \\ ${ }^{2}$ Institut de Physique et Chimie des Matériaux de Strasbourg, \\ University of Strasbourg-CNRS UMR 7504, 23 rue du Loess, F-67034 Strasbourg, France
}

\begin{abstract}
We report static and dynamic first-principles calculations that provide atomistic pictures of the initial stage of the oxidation processes occurring at the $(11 \overline{2} 0)$ surface of $4 \mathrm{H}-\mathrm{SiC}$. Our results unveil reaction pathways and their associated free-energy barriers for the adsorption of oxygen and the desorption of carbon atoms. We find that oxygen adsorption shows structural multi-stability and that the surface-bridge sites are the most stable and crucial sites for subsequent oxidation. We find that an approaching $\mathrm{O}_{2}$ molecule is adsorbed, then dissociated and finally migrates toward these surface-bridge sites with a free-energy barrier of $0.7 \mathrm{eV}$ at the $(11 \overline{2} 0)$ surface. We also find that a CO molecule is desorbed from the metastable oxidized structure upon the overcoming of a free-energy barrier of $2.4 \sim 2.6 \mathrm{eV}$, thus constituting one of the annihilation process of $\mathrm{C}$ during the oxidation. The results of the CO molecule desorption on the (11 $\overline{2} 0)$ surface are compared with the $(000 \overline{1})$ surface. A catalytic effect of dangling bonds at the surface, causing a drastic reduction of the CO desorption energy, is found on the $(000 \overline{1})$ surface and the microscopic picture of the effect is ascribed to an electron transfer from the Si to C dangling bonds. The intrinsic (11̄20) surface does not show this catalytic effect, and this is because the surface consists of an equal amount of Si and $\mathrm{C}$ dangling bonds and the electron transfer occurs before the desorption.
\end{abstract}

\section{INTRODUCTION}

Oxidation of materials is a fundamental phenomenon in nature. In particular, the oxidation of semiconductor surfaces usually leads to the formation of insulating films on the semiconductors. The resultant interface of the oxide and the semiconductor is essential in the transistor action of semiconductor devices. For instance, silicon dioxide $\left(\mathrm{SiO}_{2}\right)$ formed on $\mathrm{Si}$ is a good insulator, thus sustaining current technology.

Silicon carbide ( $\mathrm{SiC})$ is a well-known and now an emerging semiconductor material for power electronics due to its wide band-gap and the robustness under harsh environment, $\stackrel{1,2}{\Perp}$ possibly succeeding the premier $\mathrm{Si}$ in the future. Insulating films for the $\mathrm{SiC}$ device are also $\mathrm{SiO}_{2}$ formed by the thermal oxidation. However, the formation of the $\mathrm{SiO}_{2}$ films on the compound semiconductor $\mathrm{SiC}$ is a much more complicated phenomenon since oxygen reacts with both $\mathrm{Si}$ and $\mathrm{C}$, and then $\mathrm{C}$ atoms are eventually annihilated. Clarification of the microscopic mechanism for the oxidation of $\mathrm{SiC}$ is therefore extremely interesting.

$\mathrm{SiC}$ devices such as schottky diodes and field-effect transistors are already fabricated and in production 3.4 However, there still remains much room for the improvement when considering the good bulk properties of SiC: e.g., the electron mobility in the devices is typically less than $10 \%$ of the bulk value $\underline{5,6}$ This obviously comes from the poor controllability of the $\mathrm{SiO}_{2} / \mathrm{SiC}$ interface formed by the oxidation. In order to achieve the microscopic identification and then increase the controllability of the interface, intensive works have been done both experimentally ${ }^{5,7-13}$ and theoretically ${ }^{14-20}$. However, there is still no definite explanation for the deterioration mechanism of electron mobility. It is thus highly demanded to perform accurate calculations which clarifies the mechanism of the oxidation of SiC surfaces and then provides a theoretical framework to discuss the nature of the $\mathrm{SiO}_{2} / \mathrm{SiC}$ interface.

The purpose of the present paper is to focus on the initial stage of the oxidation of $\mathrm{SiC}$ and provide its atomistic reaction mechanism based on the first-principles calculations. The initial stage already includes salient features of the oxidation such as the selective oxygen attack of either $\mathrm{C}$ or $\mathrm{Si}$ atom and the $\mathrm{C}$ annihilation by plausible reaction pathways. $\mathrm{SiC}$ is a covalent and at the same time polar semiconductor and exists as various polytypes. The most stable and the commonly used is $4 \mathrm{H}-\mathrm{SiC}$ in which the atomic layers perpendicular to the bond direction stack with the quad periodicity causing the hexagonal symmetry $(4 \mathrm{H})$. We focus on the $(11 \overline{2} 0)$ surface of $4 \mathrm{H}-$ $\mathrm{SiC}$ in this paper since the devices with high electron mobility are fabricated on the surface ${ }^{21}$ Also the $(11 \overline{2} 0)$ surface is non-polar surface on which both Si and C atoms appear, being a good stage to reveal the affinity of $\mathrm{O}$ with $\mathrm{Si}$ and $\mathrm{C}$ during the oxidation process.

The initial stage of the oxidation process is decomposed into three main steps: (i) the $\mathrm{O}_{2}$ molecular adsorption and subsequent dissociation on the surface, (ii) the migration of the atomic $\mathrm{O}$ toward the formation of the $\mathrm{Si}-\mathrm{O}$ bond network, and (iii) the $\mathrm{C}$ removal from the surface as either a monoxide $\mathrm{CO}$ or a dioxide $\mathrm{CO}_{2}$. We clarify the microscopic mechanism of these processes by the static total-energy calculations and via dynamic first principles molecular dynamics simulations within the density-functional theory (DFT) ${ }^{22,23}$ framework according to the Car-Parrinello scheme (CPMD) 24 To explore the reaction pathways and obtain the corresponding free-energy barriers, we complement CPMD with the meta-dynamics 25 approach. 
The organization of this paper is as follows. Section II shows pertinent features of the present static and dynamic calculations. Results and discussion are presented in sections III, IV and V. We first present various stable and metastable atomic configurations for the oxygen adsorption obtained by the static calculations in III A and III B. Then the dynamical aspects of the $\mathrm{O}_{2}$ molecular adsorption, dissociation and the $\mathrm{O}$ migration are shown in IV A and IV B. The reaction of the carbon oxide desorption from the surface is investigated in $\mathrm{V}$ A and $\mathrm{V} B$. Section VI summarizes our findings.

\section{METHODOLOGY AND SLAB MODEL}

\section{A. Static Calculations}

Stable atomic configurations for the oxygen adsorption on the $4 \mathrm{H}-\mathrm{SiC}(11 \overline{2} 0)$ surface have been explored by the total-energy electronic-structure calculations based on DFT, with generalized gradient approximation by Perdew, Burke and Ernzerhof (PBE) ${ }^{26}$ for the exchangecorrelation energy functional. Nuclei and core electrons are treated by the projector-augmated wave (PAW) scheme by Blöchl. 27 The plane-wave basis set is used to express the Kohn-Sham orbitals and thus the electron density and the self-consistent potentials. The convergence of the basis set has been examined with bulk $\mathrm{Si}$ and $\mathrm{C}$, and molecular O. The cutoff energy of $400 \mathrm{eV}$ is found to be enough to assure the accuracy in the totalenergy difference of $2 \mathrm{meV}$ per atom. We simulate the $(1 \overline{2} 0)$ surface by the repeating slab model in which 6 atomic layers constitute the slab and each slab is separated from its adjacent images by the $15 \AA$-thick vacuum region. We use the $1 \times 2$ lateral cell which is a square of the dimension of about $10 \AA$ (see below). Correspondingly, the Brillouin zone (BZ) integration is performed by using the $3 \times 3 \times 1$ Monkhorst-Pack k-point sampling, 28 which ensures the total-energy difference of $5 \mathrm{meV}$ per cell. Computations have been done using the Vienna Ab-initio Simulation Package (VASP) $\stackrel{29}{\underline{20}}$

\section{B. Dynamic Calculations}

Dynamical aspects and thermal effects of the $\mathrm{O}_{2}$ molecular adsorption, the subsequent $\mathrm{O}$ atomic migration and eventually the $\mathrm{CO}$ molecular desorption have been accounted for by CPMD simulations complemented with the meta-dynamics approach for the sampling of the free energy landscape $33-35$ Norm-conserving pseudopotentials ${ }^{36}$ are adopted to treat the core-valence interactions for $\mathrm{H}, \mathrm{C}, \mathrm{O}$ and $\mathrm{Si}$. The PBE functional is used for the exchange-correlation interaction. The KohnSham orbitals are expanded in a plane-wave basis set with a cutoff energy of 80 Ry $(\approx 1000 \mathrm{eV})$. In all canonical simulations, the ionic temperature is controlled with Nosé-Hoover thermostat 37.38 A simulation time step of
4 a.u. ( $\approx 0.1 \mathrm{fs})$ and a fictitious electronic mass for the wave functions of 300 a.u. ensure good control of the conserved quantities along the dynamics on the ps time scale used here.

To explore the reaction pathways within the metadynamics approach, suitable reaction coordinates (collective variables, CVs) being able to account for all the slowly varying degrees of freedom have to be selected. To this aim, we choose as $\mathrm{CVs}$ the distance between the two $\mathrm{O}$ atoms of the adsorbed $\mathrm{O}_{2}$ molecule and the distance between one $\mathrm{O}$ atom and the target sites for the $\mathrm{O}_{2}$ molecular dissociation. Instead, for the $\mathrm{CO}$ molecular desorption, the coordination number ${ }^{39}$ of the $\mathrm{C}$ atom of the desorbing CO molecule is the selected CV. The expression of the coordination number is,

$$
C N_{i}=\sum_{j \neq i} \frac{1}{1+e^{k\left(d_{i j}-d^{0}\right)}}
$$

where $i$ is the index of the selected atom, $j$ runs over the surrounding atoms in the system, $d_{i j}$ is the distance between atom $i$ and atom $j, k$ determines the steepness of the decay of the analytical function shown above and $d^{0}$ is the reference distance. The index $j$ can be chosen to run over all the atoms of a specific chemical element. From the radial distribution function of $\mathrm{SiC}$ and diamond, we choose the reference distance of C-Si to be $2.2 \AA$ and C-C to be $1.8 \AA$.

\section{4H-SiC Slab Models}

$\mathrm{SiC}$ exists as various polytypes. The structural difference lies only in the stacking of atomic planes along the bond direction, i.e., the axis of the inherent three-fold rotational symmetry: e.g., the stacking sequences of $\mathrm{ABC}$ in the zincblende and $\mathrm{AB}$ in the wurtzite structures, respectively. The stacking sequence is not limited to the above two cases. Hence there are dozens of polytypes labeled by the periodicity of the stacking sequence $n$ and the symmetry (cubic or hexagonal) such as $2 \mathrm{H}$ (wurtzite), $3 \mathrm{C}$ (zincblende), $4 \mathrm{H}$ and $6 \mathrm{H}$. Among those, the $4 \mathrm{H}-\mathrm{SiC}$ is the most stable and commonly used in the device applications. Each atomic site in the $4 \mathrm{H}$ structure is inequivalent to each other and classified into two groups: the site with the locally hexagonal symmetry (h-site hereafter) and the site with the locally cubic symmetry (k-site hereafter).

On the $(11 \overline{2} 0)$ surface, in addition to the appearance of both $\mathrm{Si}$ and $\mathrm{C}$ atoms, the $\mathrm{h}$ - and $\mathrm{k}$ - sites emerge on the topmost atomic plane alternately along [0001] direction. The schematic figure of $(11 \overline{2} 0)$ surface with dangling bond direction is shown in Fig. 1

Repeating slab models are used to simulate the $\mathrm{SiC}$ surface. Adjacent slabs are isolated by the vacuum region with the thickness of $15 \AA$ to avoid the fictitious interaction between the slabs. For the $(11 \overline{2} 0)$ surface, we have used $1 \times 2$ lateral unit cell and each slab contains 6 atomic layers with the thickness of $7.5 \AA$. The bottom 


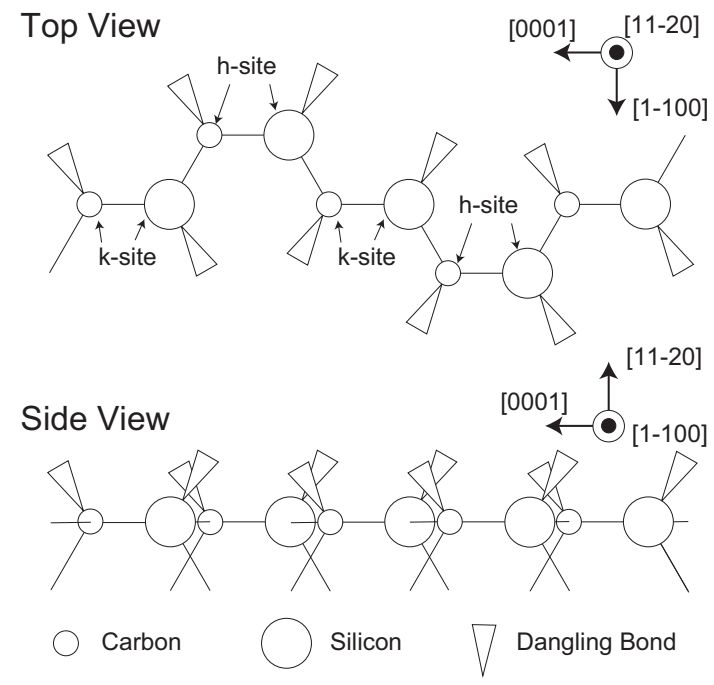

FIG. 1. Schematic picture of the surface structure of $4 \mathrm{H}-\mathrm{SiC}$ $(11 \overline{2} 0)$ with dangling bonds explicitly shown. The silicons and carbons are $\mathrm{sp}^{3}$ hybridized thus the dangling bonds on the $(11 \overline{2} 0)$ surface are not perpendicular to the surface but slightly tilted. The constituent atoms of $4 \mathrm{H}-\mathrm{SiC}$ are categorized into two kinds, h-site and k-site, as shown in the top view.
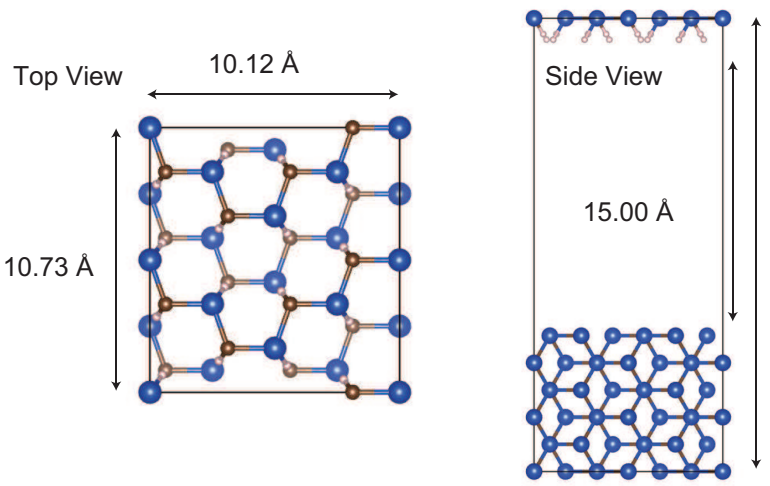

$25.84 \AA$

FIG. 2. Top view and side view of (1120) surface model. The bottom $\mathrm{SiC}$ layer and the hydrogen atoms are fixed during the geometry optimization and the CPMD simulation. The blue, brown and white balls depict $\mathrm{Si}, \mathrm{C}$ and $\mathrm{H}$ atoms, respectively.

surface is terminated by $\mathrm{H}$ atoms to remove the fictitious dangling bonds electronically. The $\mathrm{H}$ atoms and the bottom $\mathrm{SiC}$ layer are fixed during the geometrical optimization. The top view and side view of this model are shown in Fig. 2]

We also perform the calculation for the (000) surface to compare the characteristics of the initial oxidation. The adsorption sites of the $\mathrm{O}$ atom on $(000 \overline{1})$ has already been studied $\stackrel{40}{ }$ For our purpose, we use the slab model in which each slab consists of 3 atomic bilayers, i.e., 6 atomic layers with the $5.5 \AA$ thickness, with $5 \times 6$ lateral periodicity. The vacuum thickness used is $12 \AA$. The bottom surface is again terminated by $\mathrm{H}$ atoms. As there

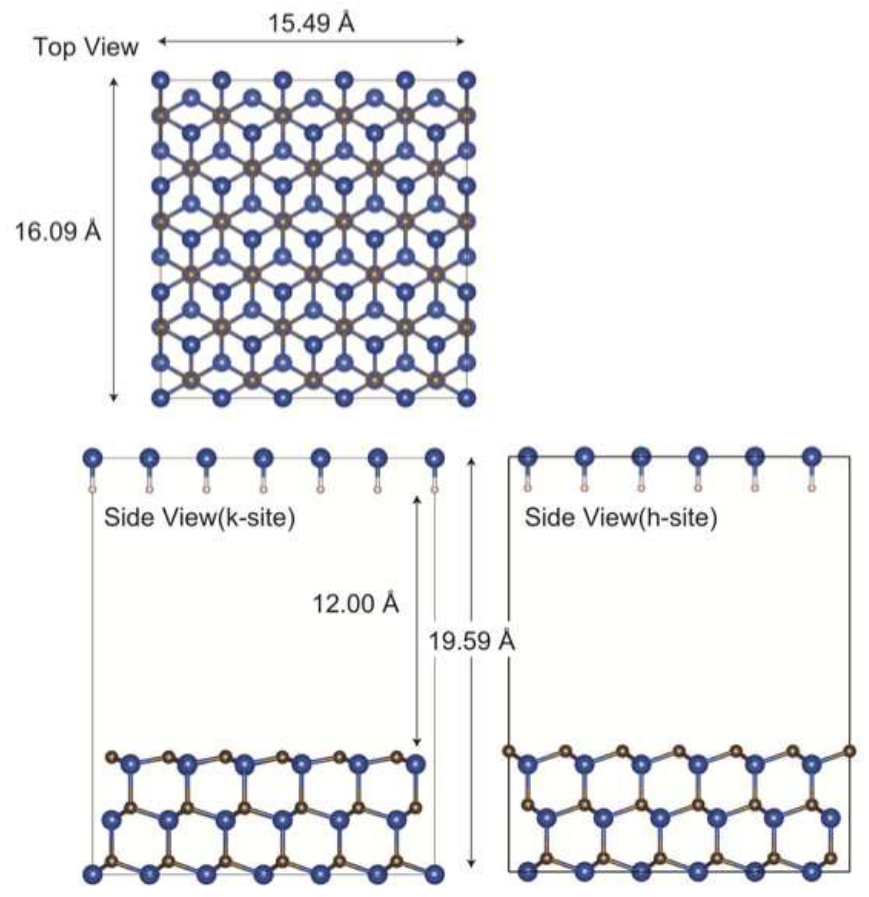

FIG. 3. Top view and side view of (0001) surface model. The bottom Si layer and hydrogen atoms are fixed during the geometry optimization and the CPMD simulation. The color code is the same as in Fig. 2

are two kinds of (0001) surface, with h-site $\mathrm{C}$ and k-site $\mathrm{C}$ as its topmost atom plane, we perform the calculation for both. The top view and side view of the slab is shown

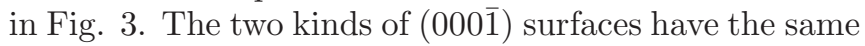
top view but the difference is in the side view.

\section{RESULT I: ADSORPTION OF O ATOMS}

In this section, we present stable and metastable structures of the oxygen adsorption on the (112 0$)$ surface of $4 \mathrm{H}-\mathrm{SiC}$ obtained by the geometry optimization in DFT. We consider the one-O-atom and the two-O-atom adsorptions. We have found various stable structures which provide the energy landscape for the oxygen adsorption.

\section{A. Single oxygen-atom adsorption}

In this subsection, we present and discuss the stable adsorption structures obtained for a single O-atom on the $(11 \overline{2} 0)$ surface. We start with the 15 initial configurations (Fig. 4), which cover all the possible adsorption sites for the one-O-atom adsorption. Those structures include all the on-top sites and bridge sites for the topmost atoms along with the bridge sites between the topmost and subsurface atoms. The surface structure of $4 \mathrm{H}-\mathrm{SiC}(11 \overline{2} 0)$ slab is relaxed before adding one oxygen atom and its atomic and electronic structures are in 
(a)

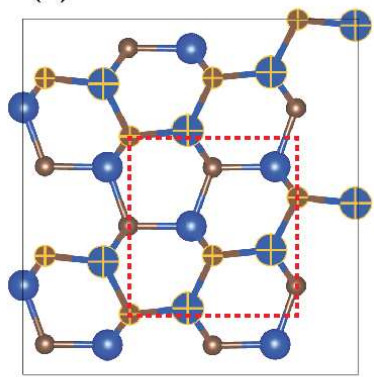

(c)

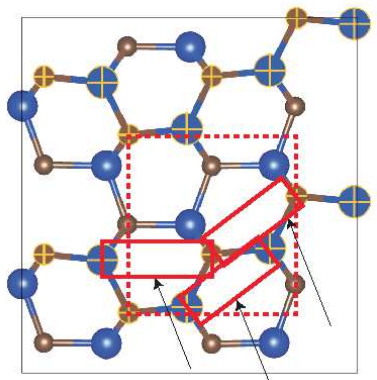

(b)

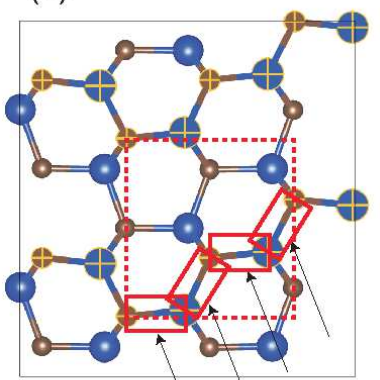

(d)

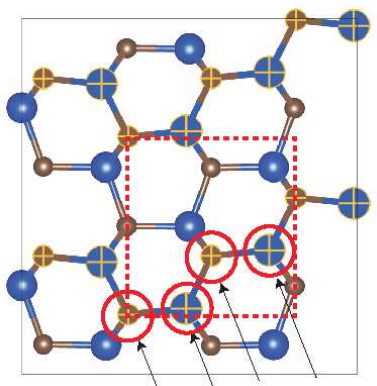

FIG. 4. Initial structures in the exploration of the single Oatom adsorption sites. The color code is the same as in Fig. 2 The dotted lines depict the primitive lateral cell. The topmost atoms are marked by the crosses. (a) clean surface. (b) 4 bridge sites between the nearest-neighbor topmost atoms. (c) 3 bridge sites between the next neighbor or next-next neighbor topmost atoms. (d) 4 on-top sites. There are also 4 bridge sites between these topmost atoms and the subsurface atoms.

good agreement with previous work by Bertelli et al $\underline{\underline{41}}$ The initial structures along with the clean-surface structure, are schematically shown in Fig. 4. After extensive geometry optimization, we have reached 10 stable and metastable adsorption structures (Fig. (5)). They are classified into (1) surface-bridge site where $\mathrm{O}$ bridges two topmost surface atoms, (2) the on-top site where $\mathrm{O}$ sits on the topmost surface atom, and (3) the subsurface-bridge site where $\mathrm{O}$ bridges the topmost surface atom and the subsurface atom.

In the surface-bridge sites, we have examined 7 possible initial configurations as in Figs. 4 (b) and (c) and have found 5 stable and metastable structures shown in Fig. 5 (a). As is clear from Fig. 11 the stable surface-bridge sites are classified into two groups: In the first group the two surface dangling bonds are directed to the bridge site and in the second they show the different directions. We call the former and the latter as the $2 \mathrm{H}$-like (labeled as 1-1-1 and 1-1-2) and the 3C-like (labeled as 1-2-1, 1-22 and 1-2-3), respectively since those configurations are the same as those on the corresponding surfaces of $2 \mathrm{H}-$ $\mathrm{SiC}$ and $3 \mathrm{C}-\mathrm{SiC}$. We have found two stable $2 \mathrm{H}$-like bridge sites shown in Fig. 5 (a): i.e., the on-bond site (labeled as 1-1-1) and the off-bond site (labeled as 1-1-2). In the on-bond site, the oxygen forms chemical bonding with $\mathrm{C}$ and $\mathrm{Si}$ atoms that are bonded with each other before

oxygen adsorption. On the other hand, in the off-bond site, the adsorption takes place on the middle of $\mathrm{C}$ and $\mathrm{Si}$ atom that are not bonded before adsorption. In these two sites, the oxygen atoms are not located right above the C-Si bond but slightly dislodged. This is due to the directions of the two surface dangling bonds. As for the 3C-like bridge sites we have found the three stable sites shown in Fig. $5(a)$ : i.e., the site between the k-site C and the k-site $\mathrm{Si}$ (labeled as 1-2-1), the site between the $\mathrm{h}$-site $\mathrm{C}$ and the k-site $\mathrm{Si}$ (labeled as 1-2-2) and the site between the $\mathrm{k}$-site $\mathrm{C}$ and the h-site $\mathrm{Si}$ (labeled as 1-2-3). In all the 3C-like bridge sites, the oxygen is located right above the $\mathrm{C}-\mathrm{Si}$ bond. We have found that bridge sites between either $\mathrm{Si}$ atoms or $\mathrm{C}$ atoms are unstable.

There are four topmost atoms inequivalent to each other on $4 \mathrm{H}-\mathrm{SiC}(11 \overline{2} 0)$ surface. Then we have examined the four on-top sites as in Fig. 4 (d). We have found that only two of the four are stable [Fig. [5 (b)]: the site on the k-site $\mathrm{Si}$ (labeled as 2-1) and the site on the h-site C (labeled as 2-2).

Each of the topmost atom participates in the two bonds on the surface and the remaining bond with the subsurface atom. Then we have examined bridge sites between the topmost and the sub-surface atoms. We have found that the three of such four sites are stable as shown in Fig. 5 (c): i.e., the subsurface-bridge sites with the topmost h-site Si (labeled as 3-1), k-site Si (labeled as 32 ) and h-site $\mathrm{C}$ (labeled as 3-3). In the subsurface-bridge sites of $4 \mathrm{H}-\mathrm{SiC}(11 \overline{2} 0)$, when the topmost atom is k-site, then subsurface atom is h-site and vice versa.

The relative stability among the adsorption structures obtained is assessed by the adsorption energy $E_{\text {ad }}$ which is defined as,

$$
E_{\mathrm{ad}}=E_{s}+\frac{1}{2} E_{\mathrm{O}_{2}}-E_{t}
$$

where $E_{t}, E_{s}$, and $E_{\mathrm{O}_{2}}$ are the total energies of the Oadsorbed surface, the clean surface and the $\mathrm{O}_{2}$ molecule, respectively. With this definition, the adsorption site with larger adsorption energy is more likely to be realized.

The calculated adsorption energies are shown in Table II We have found that the calculated adsorption energy is in the range of 0.90 to $2.35 \mathrm{eV}$, indicating that the $\mathrm{O}$-atom adsorption is the exothermic reaction. The most stable adsorption structure is achieved at the surfacebridge site. This is due to the efficient annihilation of the dangling bonds at the topmost surface atoms: At the bridge site, the $\mathrm{O}$ is capable of terminating two surface dangling bonds. The adsorption energies at the $2 \mathrm{H}$-like bridge sites are larger than those at the 3C-like bridge sites by more than a half $\mathrm{eV}$. This is due to the strains caused by the bond-angle deviation at the $3 \mathrm{C}$-like sites. For on-top sites, only the k-site $\mathrm{Si}$ and the $\mathrm{h}$-site $\mathrm{C}$ are found to be stable structures. The O-atom adsorption on the $\mathrm{C}$ on-top site is more favorable than the $\mathrm{Si}$ ontop site. This is also true for the subsurface-bridge site, indicating that the termination of $\mathrm{C}$ dangling bond is energetically more favorable. There are three sites labeled 
(a)Surface-bridge sites

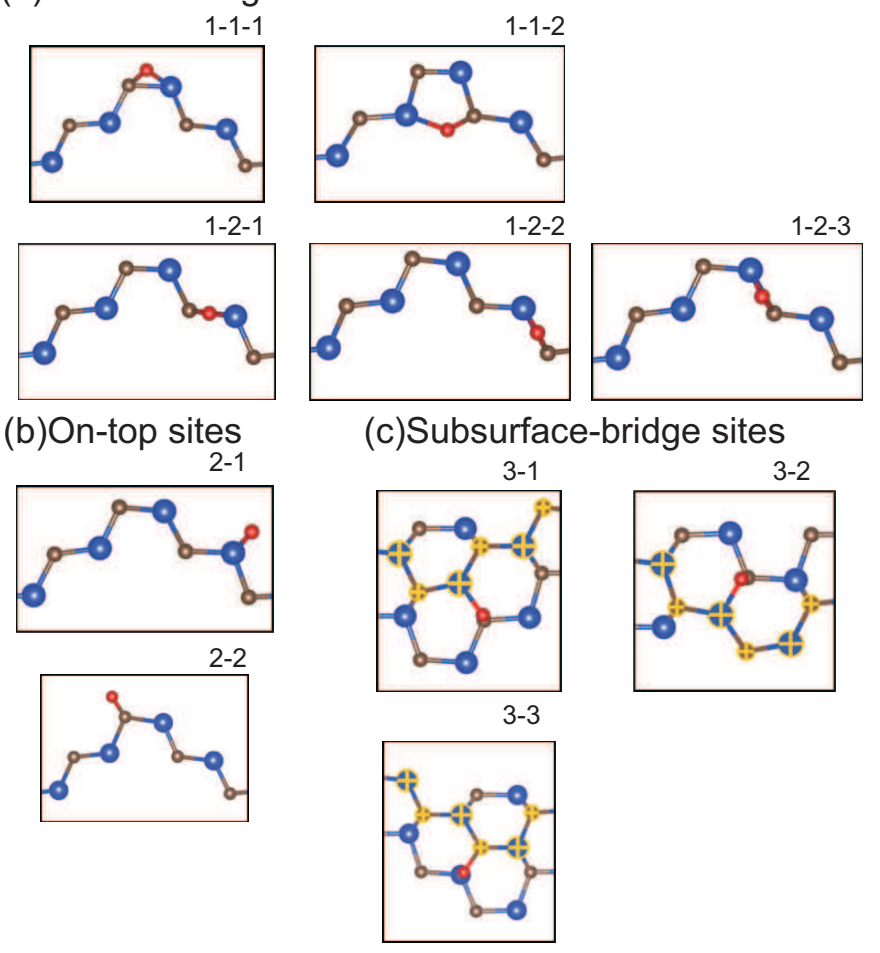

as NA in Table \. This indicates that the final structure after the geometry optimization is found to be the nearby surface-bridge sites. We have now unequivocally revealed the energy landscape for the O-atom adsorption on the $(11 \overline{2} 0)$ surface of $4 \mathrm{H}-\mathrm{SiC}$.

\section{B. Two-oxygen-atom adsorption}

In this subsection, we present the stable adsorption structures for two O-atom near to each other on the $(1 \overline{2} 0)$ surface to mimic the further oxidation at the initial stage. The plausible structure with large adsorption energy may be a pair of the most stable adsorption structures for the single $\mathrm{O}$-atom. Then we consider the $2 \mathrm{H}-$ like surface-bridge site, $1-1-1$ or 1-1-2, as a partner of such pair and seek for the plausible site for the next oxygen. After the extensive geometry optimization, we have found 9 stable pairs shown in Fig. 6 (a) as labeled from 4-1 to 4-9. A measure for the relative stability among the stable structures is the adsorption energy defined as

$$
E_{\mathrm{ad}}=E_{s}+E_{O_{2}}-E_{t} .
$$

for a single oxygen atom. (a) Two 2H-like surface-bridge sites labeled as 1-1-1 and 1-1-2, and three 3C-like surface-bridge sites labeled as 1-2-1, 1-2-2 and 1-2-3. (b) Two on-top sites labeled as 2-1 and 2-2. (c) Three subsurface-bridge sites labeled as 3-1, 3-2 and 3-3. The color code is the same as in Fig. 22 The highlighted atoms in (c) are the topmost atoms considered.

TABLE I. Adsorption energies for single O-atom at various stable sites. The superscripts for each site show the labels for the corresponding structures in Fig. 5. The NA in the table indicates that the structure have been found to be unstable, and it changed to nearby surface-bridge site after the geometry optimization.

\begin{tabular}{|c|c|c|c|c|}
\hline Sites & & & ID & $E_{\text {ad }}$ \\
\hline \multirow[t]{5}{*}{ Surface-bridge site } & \multirow[t]{2}{*}{ 2H-like } & on-bond & $1-1-1$ & $2.35 \mathrm{eV}$ \\
\hline & & off-bond & $1-1-2$ & $2.34 \mathrm{eV}$ \\
\hline & \multirow[t]{3}{*}{ 3C-like } & $\mathrm{kC}-\mathrm{kSi}$ & $1-2-1$ & $1.74 \mathrm{eV}$ \\
\hline & & $\mathrm{hC}-\mathrm{kSi}$ & $1-2-2$ & $1.68 \mathrm{eV}$ \\
\hline & & $\mathrm{kC}-\mathrm{hSi}$ & $1-2-3$ & $1.57 \mathrm{eV}$ \\
\hline \multirow[t]{4}{*}{ On-top site } & \multirow[t]{2}{*}{$\mathrm{Si}$} & h-site & NA & - \\
\hline & & k-site & $2-1$ & $1.04 \mathrm{eV}$ \\
\hline & \multirow[t]{2}{*}{$\mathrm{C}$} & h-site & $2-2$ & $1.56 \mathrm{eV}$ \\
\hline & & k-site & NA & - \\
\hline \multirow[t]{4}{*}{ Subsurface-bridge site } & \multirow[t]{2}{*}{$\mathrm{Si}$} & h-site & $3-1$ & $0.90 \mathrm{eV}$ \\
\hline & & k-site & $3-2$ & $1.21 \mathrm{eV}$ \\
\hline & \multirow[t]{2}{*}{$\mathrm{C}$} & h-site & $3-3$ & $1.27 \mathrm{eV}$ \\
\hline & & k-site & NA & - \\
\hline
\end{tabular}

The calculated adsorption energies for the 9 stable geometries are tabulated in Table I.

We have found that the adsorption energy $E_{\text {ad }}$ for the two-O-atom is generally smaller than the sum of the corresponding adsorption energies $E_{\text {sum }}$ for the single Oatom. This is due to the strain energies caused by the nearby $\mathrm{O}$ atoms. For instance, the structures having the largest sum of the adsorption energies for the single Oatom are the pairs of $2 \mathrm{H}$-like surface-bridge sites labeled 4-1 and 4-2. The calculated values for these pairs are smaller than the corresponding sum by 0.20 and $0.47 \mathrm{eV}$, respectively, due to the competing structural relaxation. Interesting exceptions are found in the structures labeled 4-8 and 4-9: i.e., the pair of the $2 \mathrm{H}$-like off-bond site 1 1-2 and the 3C-like surface-bridge site 1-2-1 of which the sum of the adsorption energies is $4.08 \mathrm{eV}$. Our structural optimization for these pairs leads to the structures (Fig. 6) in which a characteristic Si-O-C-O-Si network is formed. The calculated adsorption energy for the 4-8 is larger than the corresponding sum by $0.98 \mathrm{eV}$, and even larger than the pair 4-2 of the $2 \mathrm{H}$-like surface-bridge sites by $0.57 \mathrm{eV}$ (Table 【).

We have also examined several molecular adsorptions that have no counterparts in the single O-atom adsorplar adsorption are generally small, indicating that the $\mathrm{O}_{2}$ molecule is likely to be dissociated on the surface. However, dynamical aspects should be examined to reach a definite conclusion. tion. The calculated adsorption energies for the molecu- 
(a) Pair of one-O-atom adsorption

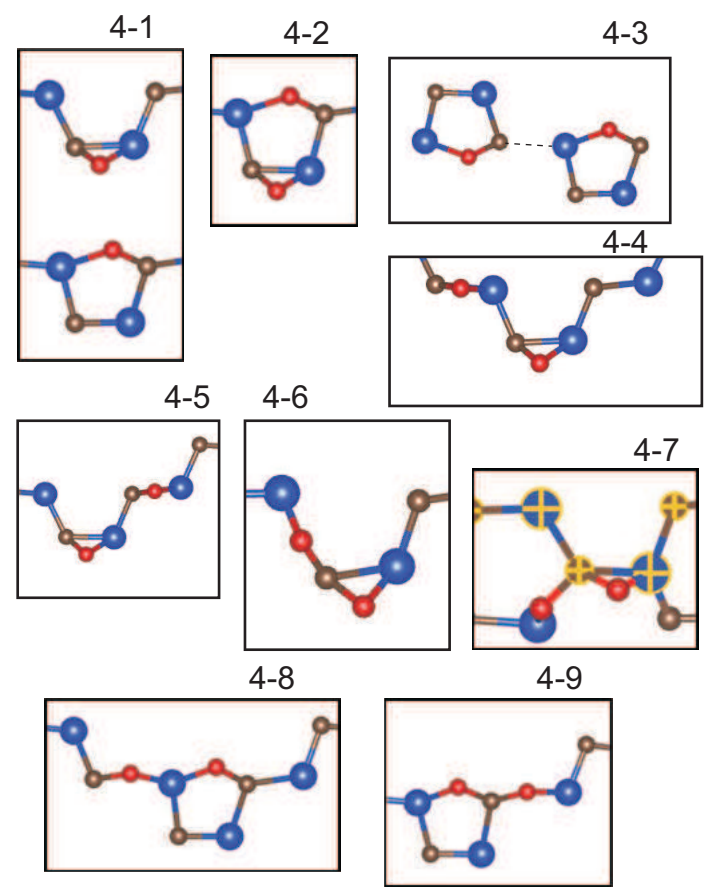

(b) Molecular adsorption

5-1

$5-2$
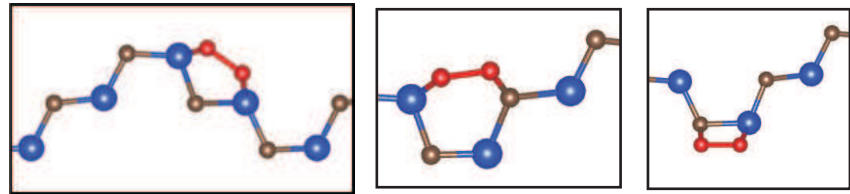

FIG. 6. Top views of stable adsorption structures for two oxygen atoms. (a) Pair-structures consisting of the stable $2 \mathrm{H}$-like adsorption site and other plausible site: The 4-1 and $4-2=(1-1-1)+(1-1-2)$, the $4-3=(1-1-2)+(1-1-2)$, the $4-4$ and $4-5=(1-1-1)+(1-2-1)$, the $4-6=(1-1-1)+(1-2-3)$ and the $4-7=(1-1-1)+(3-3)$. The $4-8$ and $4-9$, consisting of (1-12 ) and (1-2-1), are unusual structures (see text) which shows relatively large adsorption energies. (b) Molecular adsorption where the O-O bond survives after the adsorption. The color code is the same as in Fig. 2

\section{RESULT II: ADSORPTION AND DISSOCIATION OF $\mathrm{O}_{2}$ MOLECULE}

So far, We have unequivocally identified the adsorption structures in terms of their energetics for the single and two-O-atom on the basis of their relative total energies. Since these oxidation processes are highly dynamical, temperature and entropy effects should be included to get a complete insight into the oxidation phenomenon. This is the scope of the CPMD simulations done to investigate the adsorption and then dissociation of the $\mathrm{O}_{2}$ molecule on the $4 \mathrm{H}-\mathrm{SiC}(11 \overline{2} 0)$ surface. Although the ground state of an $\mathrm{O}_{2}$ molecule in the gas phase is triplet,
TABLE II. Adsorption energies $E_{\text {ad }}$ for two-O-atom at various sites. $E_{\text {sum }}$ is the sum of the adsorption energies for the respective $\mathrm{O}$ atoms forming the pair (see text).

\begin{tabular}{cccc}
\hline \hline ID & Pair & $E_{\text {sum }}$ & $E_{\text {ad }}$ \\
\hline $4-1$ & $1-1-1$ and 1-1-2 & $4.69 \mathrm{eV}$ & $4.22 \mathrm{eV}$ \\
$4-2$ & & & $4.49 \mathrm{eV}$ \\
$4-3$ & $1-1-1$ and 1-1-1 & $4.68 \mathrm{eV}$ & $4.27 \mathrm{eV}$ \\
$4-4$ & $1-1-1$ and 1-2-1 & $4.09 \mathrm{eV}$ & $3.96 \mathrm{eV}$ \\
$4-5$ & & & $3.86 \mathrm{eV}$ \\
$4-6$ & $1-1-1$ and 1-2-3 & $3.91 \mathrm{eV}$ & $3.99 \mathrm{eV}$ \\
$4-7$ & $1-1-1$ and 3-3 & $3.62 \mathrm{eV}$ & $3.95 \mathrm{eV}$ \\
$4-8$ & $1-1-2$ and 1-2-1 & $4.08 \mathrm{eV}$ & $5.06 \mathrm{eV}$ \\
$4-9$ & & & $4.66 \mathrm{eV}$ \\
\hline $5-1$ & & - & $1.93 \mathrm{eV}$ \\
$5-2$ & - & & $2.83 \mathrm{eV}$ \\
$5-3$ & & & $2.44 \mathrm{eV}$ \\
\hline \hline
\end{tabular}

we consider the approaching molecule in a spin singlet state, implying that for oxidation, the starting position of $\mathrm{O}_{2}$ is sufficiently close to the surface so that the spin polarization has already disappeared.

\section{A. $\mathrm{O}_{2}$ molecule adsorption}

In our dynamical simulations, we first equilibrate the system at room temperature $(300 \mathrm{~K})$. Then we introduce a slow oxygen molecule with a kinetic energy of $0.1 \mathrm{meV}$ in the vicinity of the surface. With this relatively small kinetic energy, the oxygen molecule approaches the energetically most favorable site among the accessible geometrical configurations, irrespective of the initial direction of the velocity. In this case, we observed that the $\mathrm{O}_{2}$ molecule first attacks the k-site $\mathrm{Si}$ and then the nearby h-site Si to form a Si-O-O-Si bridge structure (Fig. 7). As this process occurs spontaneously without imposing any constraints to drive a specific reaction, the energy barrier for the adsorption of the single $\mathrm{O}_{2}$ molecule to $4 \mathrm{H}-\mathrm{SiC}(11 \overline{2} 0)$ should have as an upper bound value of $25.7 \mathrm{meV}$, i.e. the system temperature at $300 \mathrm{~K}$. In the $\mathrm{Si}-\mathrm{O}-\mathrm{O}-\mathrm{Si}$ bridge structure, the $\mathrm{O}-\mathrm{O}$ distance is stretched beyond the bond length of the $\mathrm{O}_{2}$ molecule. However, our analysis of the electron density indicates clearly that the chemical bond is preserved. Therefore the molecular adsorption occurs on the $(11 \overline{2} 0)$ surface at the initial stage of the process and this adsorption does not result in a molecular dissociation. The structure realized is the same as 5-1 in Fig. 6. Moreover, these dynamical simulations indicate a higher affinity of Si atoms than $\mathrm{C}$ atoms to the $\mathrm{O}$. 


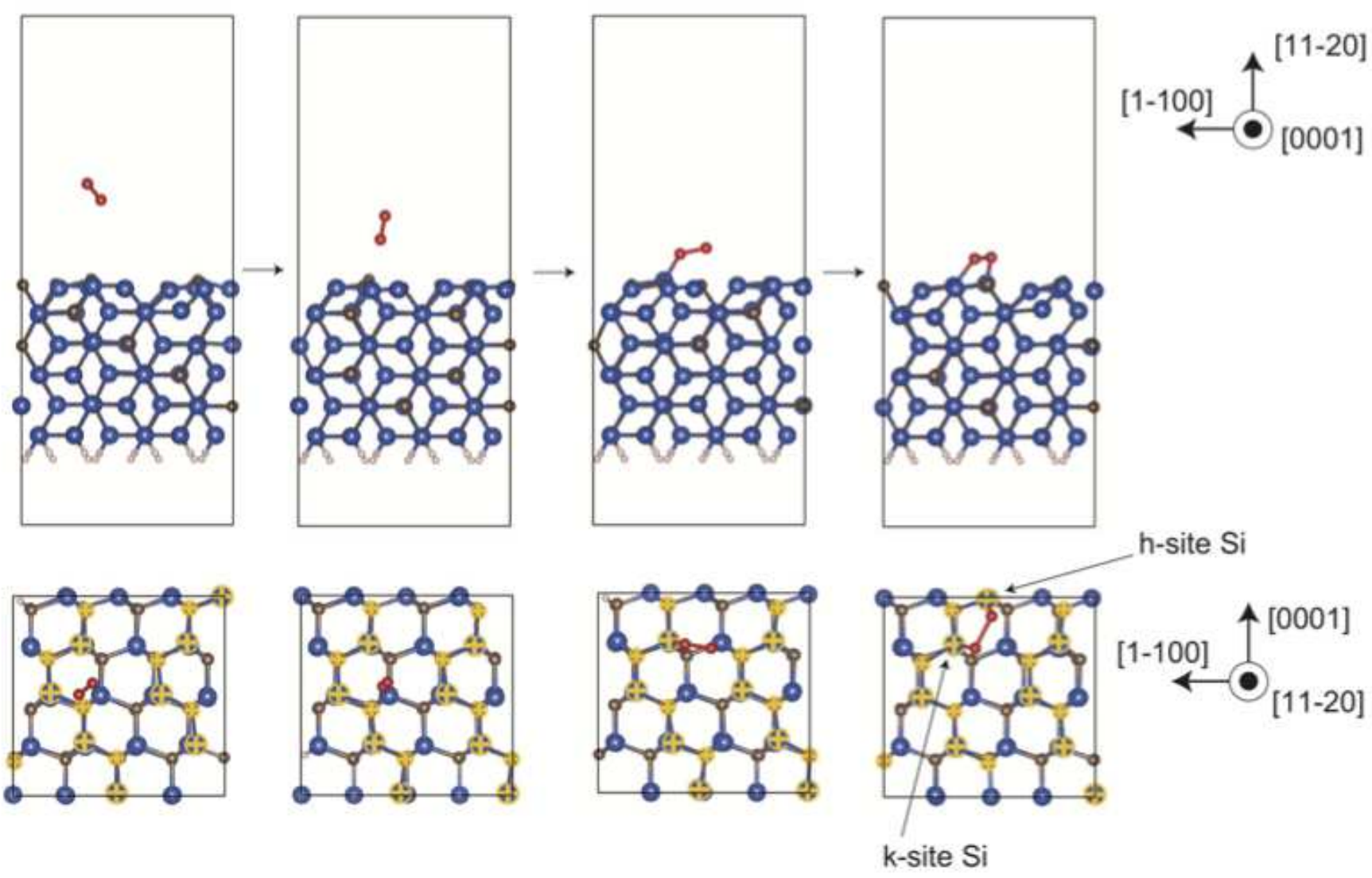

FIG. 7. Adsorption of an $\mathrm{O}_{2}$ molecule to $(11 \overline{2} 0)$ surface represented by snapshots during the CPMD simulations. Side views (upper panels) and top views (lower panels). When the velocity of $\mathrm{O}_{2}$ molecule toward the surface is sufficiently small, the molecule is adsorbed on the surface forming Si-O-O-Si structure. The color code is the same as in Fig. 2

\section{B. $\mathrm{O}_{2}$ dissociation}

In the previous subsection, we have clarified that the molecular $\mathrm{O}_{2}$ adsorption takes place on the $(11 \overline{2} 0)$ surface at room temperature. On the other hand, our DFT calculations in section III show that the adsorption energy for an $\mathrm{O}_{2}$ molecule is much smaller than the dissociative adsorption of two-O-atom (Table III). Then the dissociation of $\mathrm{O}_{2}$ is expected to occur and this process is indispensable in order for the oxidation to proceed. This is very different from the adsorption of $\mathrm{O}_{2}$ molecule on $4 \mathrm{H}-\mathrm{SiC}(000 \overline{1})$ surface where the dissociation occurs spontaneously ${ }_{\underline{42}}$ We have adopted the final structure obtained from the CPMD simulation for the $\mathrm{O}_{2}$ molecular adsorption in the preceding subsection as an initial structure, and then conducted further CPMD simulations complemented with the meta-dynamics approach for the sampling of the reaction pathways and to work out corresponding free-energy barriers for the dissociation. The selected CVs were the O-O distance and the distance between an $\mathrm{O}$ atom and a $\mathrm{C}$ atom at the $\mathrm{k}$-site. We have found that the $\mathrm{O}_{2}$ molecule is dissociated and the resulting $\mathrm{O}$ atoms are adsorbed at the on-top sites of the h-site $\mathrm{Si}$ and the k-site $\mathrm{Si}$, respectively. The ac- tivation barrier for this $\mathrm{O}_{2}$ dissociation is $0.73 \mathrm{eV}$ (Fig. 8). From the DFT calculations for the single $\mathrm{O}$ atom adsorption in the previous section, the $\mathrm{O}$ atom at the on-top site of the h-site $\mathrm{Si}$ is unstable (Table \). Considering that the $2 \mathrm{H}$-like on-bond bridge structure 1-1-1 is reached by small dislodgment from the on-top site on the h-site Si [Fig. [5 (a)] and the 1-1-1 structure is the most stable adsorption site, the $\mathrm{O}$ atom at the on-top site on the h-site $\mathrm{Si}$ after the $\mathrm{O}_{2}$ dissociation transforms to the nearby bridge structure 1-1-1 with no appreciable energy barrier. As for the $\mathrm{O}$ atom at the on-top site on the k-site $\mathrm{Si}$ which is metastable, we have performed CPMD simulations and found that it transforms to another $2 \mathrm{H}$-like off-bond bridge site 1-1-2 with the activation energy of $0.13 \mathrm{eV}$ (Fig. 8).

Dynamical simulations presented in this section, along with the static calculations in the preceding section III, clarify that oxygen is adsorbed in an undissociated molecular form at the beginning of the process and then dissociated toward more stable atomic-oxygen adsorption structures upon the overcoming of a free-energy barrier of $1 \mathrm{eV}$ or less. The results also reflect the higher oxygen affinity of Si than $\mathrm{C}$ atoms. We have found several stable structures for the pair of the resultant $\mathrm{O}$ atoms. This 


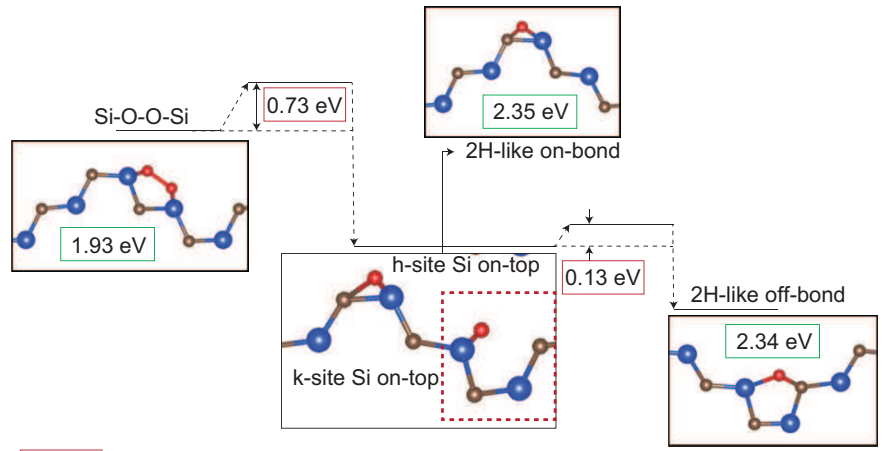

Free-energy barrier by meta-dynamics

Adsorption energy by DFT

FIG. 8. Structural transformation during $\mathrm{O}_{2}$ dissociation at $(11 \overline{2} 0)$ surface. Starting from $\mathrm{O}_{2}$ molecular adsorption structure (left panel), the $\mathrm{O}-\mathrm{O}$ bond breaks with $0.73 \mathrm{eV}$ free energy barrier (middle bottom panel). The h-site Si on-top site is not stable for $\mathrm{O}$ atom so that it is transformed into $2 \mathrm{H}$ like on-bond structure (middle top panel). It takes $0.13 \mathrm{eV}$ for the $\mathrm{k}$-site $\mathrm{Si}$ on-top structure to go to more stable $2 \mathrm{H}$-like off-bond structure (right panel).

multi-stability is the characteristics at the initial stage of the oxidation on $(11 \overline{2} 0)$ surface.

\section{RESULT III: DESORPTION OF CO MOLECULE}

The remaining important process in the initial stage of the oxidation of $\mathrm{SiC}$ is the removal of carbon atoms by specific reactions, which is a necessary step for $\mathrm{SiO}_{2}$ films to be formed. By examining the stable structures carrying a single $\mathrm{O}$-atom and two-O-atom discussed in the previous sections [Figs. 5 and [6, we consider the desorption of a $\mathrm{CO}$ molecule from the surface as one of the important reaction process for the $\mathrm{C}$ removal. To this aim, we resort to CPMD simulation to inspect the desorption of $\mathrm{CO}$ and we compare this $\mathrm{CO}$ desorption on the $(11 \overline{2} 0)$ surface with the analogous process on the $(000 \overline{1})$ surface to illustrate the peculiarities of the $(11 \overline{2} 0)$ surface.

We examine the $\mathrm{CO}$ desorption on the oxygen adsorbed $\mathrm{SiC}$ surfaces with and without hydrogen termination. This is motivated by the interest in the role of the surface dangling bonds in the desorption process on one hand. On the other, the H-terminated surface mimics the $\mathrm{SiO}_{2} / \mathrm{SiC}$ interface where the $\mathrm{Si}-\mathrm{O}-\mathrm{C}$ network is likely to exist during the oxidation with the dangling bonds being not near to the network.

\section{A. CO desorption from the clean surface}

Among the stable and metastable oxygen adsorbed structures, the structure labeled $2-2$ in Fig. 5] is the
TABLE III. Desorption energy barriers for CO on cleavage surface.

\begin{tabular}{ccc}
\hline \hline Surface orientation & $(11 \overline{2} 0)$ & $(000 \overline{1})$ \\
\hline h-site & $0.59 \mathrm{eV}$ (Migration) & $1.51 \mathrm{eV}$ \\
k-site & $2.54 \mathrm{eV}$ & $1.68 \mathrm{eV}$ \\
\hline \hline
\end{tabular}
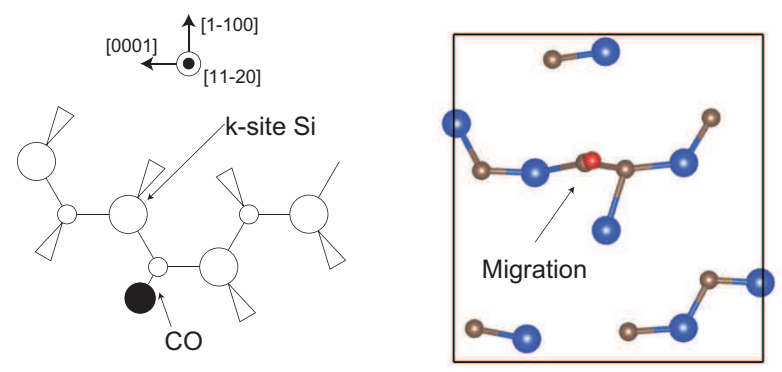

FIG. 9. (Left panel) Schematic view of the configuration of the dangling bonds. (Right panel) Top view of the structure after the migration of $\mathrm{CO}$ at the h-site on $(11 \overline{2} 0)$ surface.

most probable candidate structure for the $\mathrm{CO}$ desorption. As stated in section II, we have chosen the coordination number of the target $\mathrm{C}$ atom as the collective variable, i.e., the reaction coordinate, in the CPMD simulations with the meta-dynamics. We have found that the desorption process on the $(000 \overline{1})$ is just the elongation of the $\mathrm{C}$-Si distance, leading to the CO desorption. The calculated free-energy barriers are $1.51 \mathrm{eV}$ and $1.68 \mathrm{eV}$ for the h-site and k-site carbon respectively. As for the (112 0 ) surface, we have found that the desorption of CO takes place for the k-site $\mathrm{C}$. The calculated free-energy barrier is $2.54 \mathrm{eV}$, which is much higher than that on the $(000 \overline{1})$ surface (Table III). As for the h-site, we have found that the free-energy barrier for the surface migration of the $\mathrm{CO}$ is much lower than the desorption. We have found that the $\mathrm{CO}$ unit at the h-site migrates to the bridge site shown in Fig. 9] The obtained free-energy barrier is $0.59 \mathrm{eV}$. This relatively low energy barrier is caused by the surface dangling bonds at the k-site Si (Fig. 9). It is noteworthy that this migration process on the $(11 \overline{2} 0)$ surface corresponds to the outward migration of the $\mathrm{CO}$ unit for the $(000 \overline{1})$ surface of $\mathrm{SiC}$, which may assists in the carbon annihilation from the interface.

\section{B. CO Desorption from the H-terminated surface}

We next consider the CO desorption from the hydrogen terminated oxidized surface. The energy barriers obtained by our meta-dynamics-enhanced CPMD simulations are shown in Table IV Specifically, the free-energy barriers for the desorption from the $(11 \overline{2} 0)$ surface are 2.61 and $2.41 \mathrm{eV}$ for h-site and k-site respectively. For comparison, on the $(000 \overline{1})$ surface the calculated values are 2.62 and $2.59 \mathrm{eV}$, respectively. The free-energy bar- 
TABLE IV. Desorption energy barriers for $\mathrm{CO}$ on $\mathrm{H}-$ terminated surface.

\begin{tabular}{ccc}
\hline \hline Surface orientation & $(11 \overline{2} 0)$ & $(000 \overline{1})$ \\
\hline h-site & $2.61 \mathrm{eV}$ & $2.62 \mathrm{eV}$ \\
k-site & $2.41 \mathrm{eV}$ & $2.59 \mathrm{eV}$ \\
\hline \hline
\end{tabular}

riers for the $\mathrm{CO}$ desorption from a H-terminated surface are rather similar on both surfaces and site type, either hor k-sites. It is noteworthy that the obtained values are also close to the desorption barriers on the $\mathrm{H}$-free $(11 \overline{2} 0)$ surface (Table III). It is also remarkable that the desorption barriers from the $\mathrm{H}$-free $(000 \overline{1})$ surface are definitely smaller, about one and a half $\mathrm{eV}$. We have found that this trend in the desorption barriers is parallel to the trend in the total energies of the final geometries.

By analyzing the electronic structures, we have found that this trend is caused by the presence or absence of the partially filled $\mathrm{C}$ dangling bonds. For the desorption from the H-free $(000 \overline{1})$ surface, three Si dangling bonds are generated after the $\mathrm{CO}$ desorption. Each electron in the Si dangling bonds are transferred to the dangling bonds of the topmost $\mathrm{C}$ atoms which are located in energy below the Si dangling bond ( $\mathrm{C}$ has larger electron affinity than $\mathrm{Si}$ ) and partially filled, leading to the surface states. This electronic energy gain is the reason for the lower finalstate and transition-state energy. For the $(11 \overline{2} 0)$ surface, there exist an equal amount of both $\mathrm{Si}$ and $\mathrm{C}$ dangling bonds. Each electron of the Si dangling bond is already transferred to the $\mathrm{C}$ dangling bond, leading to the gap of the surface states, before the CO desorption. Then it is obvious that the electronic energy gain is absent. For the H-terminated surface, this mechanism of the electron acceptance by the $\mathrm{C}$ dangling bonds and the resultant energy gain is invalid. We just observe the structural relaxation of the two of the three topmost Si atoms after the $\mathrm{CO}$ desorption on the (000) $)$ surface, which is minor in the energetics.

\section{CONCLUSION}

We have performed static and dynamic calculations based on DFT to reveal microscopic mechanisms of oxidation of $\mathrm{SiC}(11 \overline{2} 0)$ surface at its initial stage.

Firstly, we have identified stable and metastable adsorption structures for a single oxygen atom and two oxygen atoms. This was done by exhaustive search for possible geometries by the static DFT calculations. We have found 10 stable structures for the single oxygen atom and 12 stable structures for the two oxygen atoms. From the calculated adsorption energies, we have found that the two particular surface-bridge sites where the local configurations are wurtzite-like (2H-like) are the most stable adsorption sites for the single oxygen atom. Other surface-bridge sites where the local structures are zincblende-like (3C-like) are found to be the next stable adsorption sites. We have also found that the on-top sites and the subsurface-bridge sites show relatively small adsorption energies. The energetics obtained shows that oxygen tends to attack the carbon, since the termination of carbon dangling bonds is energetically more favorable. For the two-oxygen-atom adsorption, we have found that the pairs of the surface-bridge sites are the most stable adsorption sites. Yet the adsorption energies are generally smaller than the sum of the adsorption energies at the surface-bridge sites for the single $\mathrm{O}$ atom due to the strains caused by the insertion of $\mathrm{O}$ atoms. Interesting exception is the pair of the $2 \mathrm{H}$-like off-bond surfacebridge site and the 3C-like surface-bridge site. This configurations are composed of the -C-O-Si-O-C or -Si-O-CO-Si- network along [0001] direction, thus releasing the strain energy. Our results are indicative of the importance of the surface-bridge sites in the initial stage of the oxidation process. In addition to that, our finding of multi-stability of the adsorption structures is the characteristics of the high-Miller index surface like the (11 $\overline{2} 0)$ surface where various sites with different symmetries provide comparable accommodation sites for the oxygen.

Secondly, we have performed CPMD simulations to inspect reaction pathways and to compute the corresponding free-energy barriers for the adsorption and dissociation of the oxygen molecule and also the desorption of the CO molecule from the $(11 \overline{2} 0)$ surface. The free-energy barriers have been obtained by the CPMD simulations combined with the meta-dynamics. We have found that the $\mathrm{O}_{2}$ molecule is adsorbed on the surface-bridge site where each of the $\mathrm{O}$ atom forms a bond with the topmost $\mathrm{Si}$ atom. This shows higher affinity of Si than $\mathrm{C}$ with $\mathrm{O}$ dynamically. Our calculation also shows that the adsorption energy of the $\mathrm{O}_{2}$ molecule is much smaller that that of $\mathrm{O}$ atoms. Our CPMD calculations have indeed revealed that the adsorbed $\mathrm{O}_{2}$ molecule is dissociated with the free-energy barrier of $0.7 \mathrm{eV}$ and the resultant two $\mathrm{O}$ atoms are eventually adsorbed at the $2 \mathrm{H}$-like surfacebridge sites.

The desorption of the $\mathrm{CO}$ molecule is the fundamental process in the annihilation of $\mathrm{C}$ during the oxidation. We have performed the CPMD simulations for the CO desorption from the $(11 \overline{2} 0)$ surface and also from the $(000 \overline{1})$ surface for comparison. We have found that $\mathrm{C}$ is removed in the form of $\mathrm{CO}$ molecule. The calculated free-energy barriers for the desorption are $2.4 \sim 2.6 \mathrm{eV}$ for most cases, and this is attributed to be the free-energy needed to break three Si-C bonds. We have examined both the clean and the hydrogen-terminated oxidized surface to clarify the role of the surface dangling bonds in the CO desorption. We have found that the dangling bonds at the surface play an important role during the oxidation; they can reduce the activation barrier for certain reactions and can also change the dominant reaction pathways. For the $(000 \overline{1})$ surface, an interesting reduction of the desorption barrier by about $1 \mathrm{eV}$ is observed. Our analyses have clarified that this reduction is caused by 
the electron transfer from the Si dangling bonds to the $\mathrm{C}$ dangling bonds upon the CO desorption. For the (112 0$)$ surface, a change from desorption of CO to surface migration occurs when dangling bonds are positioned near the CO. Our findings about the dangling-bond effects are crucial when constructing further oxidized theoretical $\mathrm{SiO}_{2} / \mathrm{SiC}$ interface model to study the chemical reactions at the interface. The dangling bonds at the interface need to be treated carefully because they can enhance certain reactions.

\section{ACKNOWLEDGMENTS}

This work was partly supported by the project for Priority Issue "Creation of new functional devices and highperformance materials to support next-generation indus- tries)" to be tackled by using Post-K Computer, conducted by Ministry of Education, Culture, Sports, Science and Technology, Japan, and by the JSPS KAKENHI Grant Number 15H03601. Computations were performed at the Supercomputer Center at the Institute for Solid State Physics, The University of Tokyo, The Research Center for Computational Science, National Institutes of Natural Sciences, the Center for Computational Science, University of Tsukuba, and at the HPC Mesocenter (Equip@Meso) of the University of Strasbourg, France. H. L. was supported by Japan Society for the Promotion of Science through Program for Leading Graduate Schools (MERIT). M. B. acknowledges LaBex "Nanoparticles Interacting with their Environment" ANR-11-LABX-0058_NIE and GENCI under allocation x2016096092.
${ }^{1}$ R. G. Azevedo, D. G. Jones, A. V. Jog, B. Jamshidi, D. R. Myers, L. Chen, X. a. Fu, M. Mehregany, M. B. J. Wijesundara, and A. P. Pisano, IEEE Sensors Journal 7, 568 (2007)

2 M. T. Soo, K. Y. Cheong, and A. F. M. Noor, Sensors and Actuators B: Chemical 151, 39 (2010)

3 J. Yu, S. J. Ippolito, M. Shafiei, D. Dhawan, W. Wlodarski, and K. Kalantar-zadeh, Applied Physics Letters 94, 013504 (2009).

4 M. K. Das, C. Capell, D. E. Grider, S. Leslie, J. Ostop, R. Raju, M. Schutten, J. Nasadoski, and A. Hefner, in 2011 IEEE Energy Conversion Congress and Exposition (2011) pp. 2689-2692.

${ }^{5}$ M. Yoshikawa, K. Inoue, H. Seki, Y. Nanen, M. Kato, and T. Kimoto, Applied Physics Letters 102, 051612 (2013).

${ }^{6}$ M. R. Werner and W. R. Fahrner, IEEE Transactions on Industrial Electronics 48, 249 (2001)

7 T. Zheleva, A. Lelis, G. Duscher, F. Liu, I. Levin, and M. Das, Applied Physics Letters 93, 022108 (2008)

8 S. Wang, S. Dhar, S.-r. Wang, A. C. Ahyi, A. Franceschetti, J. R. Williams, L. C. Feldman, and S. T. Pantelides, Phys. Rev. Lett. 98, 026101 (2007)

9 Y. Song and F. W. Smith, Applied Physics Letters 81 (2002).

10 T. Seyller, R. Graupner, N. Sieber, K. V. Emtsev, L. Ley, A. Tadich, J. D. Riley, and R. C. G. Leckey, Phys. Rev. B 71, 245333 (2005)

11 D. Goto, Y. Hijikata, S. Yagi, and H. Yaguchi, Journal of Applied Physics 117, 095306 (2015)

12 R. Heihachiro Kikuchi and K. Kita, Applied Physics Letters 104, 052106 (2014)

13 K. Kita, R. H. Kikuchi, and H. Hirai, ECS Transactions 61, 135 (2014).

14 A. Ito, T. Akiyama, K. Nakamura, T. Ito, H. Kageshima, M. Uematsu, and K. Shiraishi, Japanese Journal of Applied Physics 54, 101301 (2015)

15 C. J. Kirkham and T. Ono, Journal of the Physical Society of Japan 85, 024701 (2016).

16 R. Buczko, S. J. Pennycook, and S. T. Pantelides, Phys. Rev. Lett. 84, 943 (2000).

17 A. Gavrikov, A. Knizhnik, A. Safonov, A. Scherbinin,
A. Bagaturyants, B. Potapkin, A. Chatterjee, and K. Matocha, Journal of Applied Physics 104, 093508 (2008).

18 T. Ono and S. Saito, Applied Physics Letters 106, 081601 (2015)

19 T. Akiyama, A. Ito, K. Nakamura, T. Ito, H. Kageshima, M. Uematsu, and K. Shiraishi, Surface Science 641, 174 (2015)

20 W. Li, J. Zhao, Q. Zhu, and D. Wang, Applied Physics Letters 103, 211603 (2013).

21 T. Kimoto, Y. Kanzaki, M. Noborio, H. Kawano, and H. Matsunami, Japanese Journal of Applied Physics 44, 1213 (2005)

22 P. Hohenberg and W. Kohn, Phys. Rev. 136, B864 (1964)

${ }^{23}$ W. Kohn and L. J. Sham, Phys. Rev. 140, A1133 (1965).

24 R. Car and M. Parrinello, Phys. Rev. Lett. 55, 2471 (1985).

25 A. Laio and M. Parrinello, PNAS 99, 12562 (2002)

26 J. P. Perdew, K. Burke, and M. Ernzerhof, Phys. Rev. Lett. 77, 3865 (1996).

27 P. E. Blöchl, Phys. Rev. B 50, 17953 (1994).

${ }^{28} \mathrm{H}$. J. Monkhorst and J. D. Pack, Phys. Rev. B 13, 5188 (1976)

29 G. Kresse and J. Hafner, Phys. Rev. B 47, 558 (1993)]

30 G. Kresse and J. Hafner, Phys. Rev. B 49, 14251 (1994).

31 G. Kresse and J. Furthmüller, Computational Materials Science 6, 15 (1996).

32 G. Kresse and J. Furthmüller, Phys. Rev. B 54, 11169 (1996).

33 D. Marx and J. Hutter, "Ab-initio molecular dynamics: Theory and implementation," in Modern Methods and Algorithms of Quantum Chemistry, NIC, edited by J. Grotendorst (Forschungszentrum Jülich, 2000) Chap. 13, pp. 301-449, i ed.

${ }^{34} \mathrm{~W}$. Andreoni and A. Curioni, Parallel Computing 26, 819 (2000).

35 D. Marx and J. Hutter, Ab Initio Molecular Dynamics (Cambridge University Press, 2009).

36 N. Troullier and J. L. Martins, Phys. Rev. B 43, 1993 (1991).

37 S. Nosé, The Journal of Chemical Physics 81 (1984).

38 W. G. Hoover, Phys. Rev. A 31, 1695 (1985).

39 M. Sprik, Faraday Discuss. 110, 437 (1998). 
40 J. Wang, L. Zhang, Q. Zeng, G. L. Vignoles, and L. Cheng, Journal of Physics: Condensed Matter 22, 265003 (2010)

41 M. Bertelli, J. Homoth, M. Wenderoth, A. Rizzi, R. G. Ulbrich, M. C. Righi, C. M. Bertoni, and A. Catellani,
Phys. Rev. B 75, 165312 (2007)

42 Y. Matsushita and A. Oshiyama, (unpublished). 